\title{
Electron Backscatter Diffraction of Nuclear Materials
}

\author{
Daniel Jädernäs ${ }^{1}$, Pia Tejland ${ }^{1}$ \\ ${ }^{1}$ Studsvik Nuclear AB, Material Technology - Hot Cell Laboratory, Nyköping, Sweden.
}

Studying and understanding the behavior and microstructure of nuclear materials, many of them subjected to neutron radiation, is critical for the safe and continued operation of nuclear power plants. One important tool for microstructural studies is scanning electron microscopy (SEM) in combination with different analytical techniques, such as wavelength and energy dispersive $\mathrm{x}$-ray spectroscopy (WDS, EDS) and electron backscatter diffraction (EBSD) analysis. Characterization of irradiated materials is though greatly complicated by the radioactivity of the materials. The sample preparation often requires a shielded hot cell environment, the volume of material needs to be reduced in order to handle the samples outside a hot cell and the SEMs often need custom built sample transfer devices and additional shielding to protect the operator. Furthermore, modern electronic components are often sensitive to radiation, making shielding of these components necessary. These factors have led to a delay in the application of advanced microscopy techniques for nuclear applications. However, during the last couple of years, several nuclear laboratory facilities have realized the benefit of such analyses, and invested in more advanced analytical tools. This work focuses on EBSD on irradiated nuclear structural materials (e.g. steels, welds and Ni-based alloys) and nuclear fuel $\left(\mathrm{UO}_{2}\right)$.

The dual column focused ion beam (FIB) and field emission gun SEM used in the work presented here is a Zeiss Auriga Cross Beam system. The instrument is equipped with EDS (Apollo X) and WDS (TEXS) systems as well as an EBSD camera (Hikari), all supplied by EDAX for elemental analysis and structural material characterization. A custom built airlock is installed for safe transfer of radioactive samples and a lead wall separates the operator from the instrument. The instrument is placed in a controlled area at Studsvik's hot cell laboratory, and consequently radioactive materials can be examined. All EBSD work was performed using $20 \mathrm{kV}$ electron acceleration voltage, $\sim 10 \mathrm{nA}$ beam current and a working distance of $14 \mathrm{~mm}$. High resolution EBSD work was performed using smaller condenser apertures resulting in lower probe current. The sample tilt was $70^{\circ}$. In some cases, a surface prepared using the FIB or electrolytic polishing was used to improve diffraction pattern quality.

The microstructural evolution of the $\mathrm{UO}_{2}$ fuel pellets during operation in a nuclear power reactor is important to understand in order to anticipate its operating limits. EBSD is a useful tool to help understanding these limiting factors, e.g. fuel swelling, fission product gas release and aggressive species release to the cladding (assisting in stress corrosion cracking of the $\mathrm{Zr}$-alloy cladding). All these can be related to the microstructure of the material. $\mathrm{UO}_{2}$ has a face centered cubic $\left(\mathrm{CaF}_{2}\right)$ crystal lattice and it has been shown to be relatively straight forward to perform EBSD also on irradiated $\mathrm{UO}_{2}$, despite the high degree of irradiation damage accumulated in the material ( $\sim 1 \mathrm{dpa}$ /day). Figure 1 shows a radial profile backscattered electron (BSE) SEM micrograph of a large grained $\mathrm{UO}_{2}$ fuel type. EBSD helps understanding the nucleation of fission gas bubbles, subgrain formation and grain boundary formation in different regions of the pellet and also gives basic information like grain size distribution. These features control the fission gas release and transport of fission products. An example is shown in Figure 2. A BSE micrograph of the rim region shows the subgrain structure and EBSD gives further information about the microstructure, e.g. that the position of fission gas bubbles are in the subgrain boundaries and also that most of the subgrain boundaries are high angle boundaries. 
Low alloyed steel/austenitic weld/stainless steel joints are important structural components of nuclear power reactors. It is crucial to test and understand their mechanical properties, and detailed knowledge of the microstructural state of these components is needed to fully interpret the results of such testing. Apart from knowing the grain size, phase composition and texture etc., it is also of interest to know how much deformation that has been introduced in the material during manufacturing and operation. Figure 3 shows EBSD inverse pole figure maps of each region of such a joint and a graph showing the strain distribution traversing the weld. The residual strain is interpreted as being proportional to the amount of lattice rotation evaluated from the EBSD measurements. It can be observed that the strain increases towards the weld material from both the stainless steel and the low alloyed steel sides while the maximum strain position is located close to the center of the weld.

In summary, this work focuses on EBSD characterization of different types of irradiated material and demonstrates the strengths and difficulties using this technique.

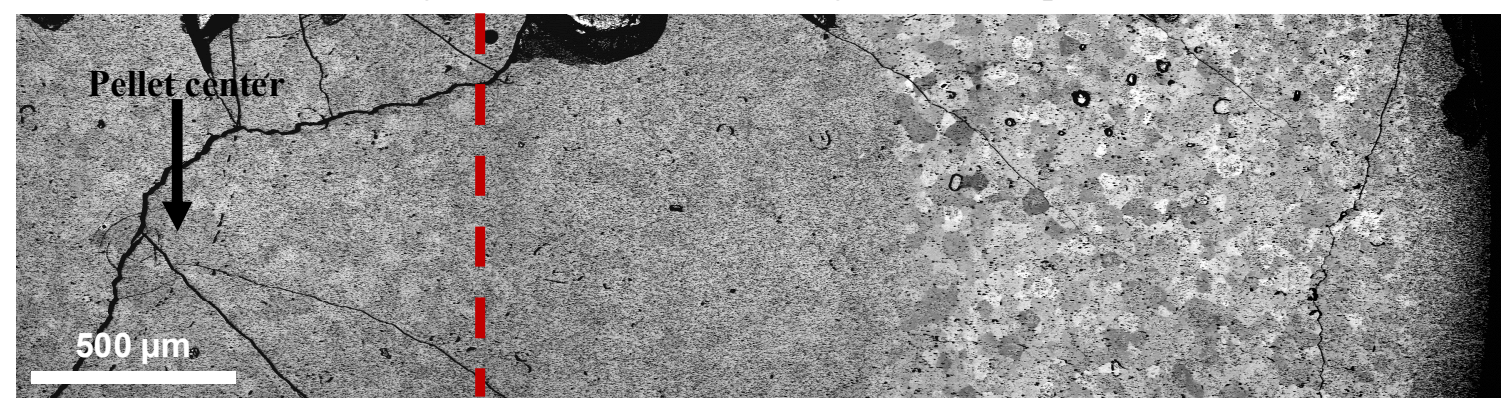

Figure 1. Large-grained irradiated $\mathrm{UO}_{2}$ fuel pellet radial overview BSE micrograph showing regions having different microstructural features. Red arrow shows the rim region in the pellet periphery.
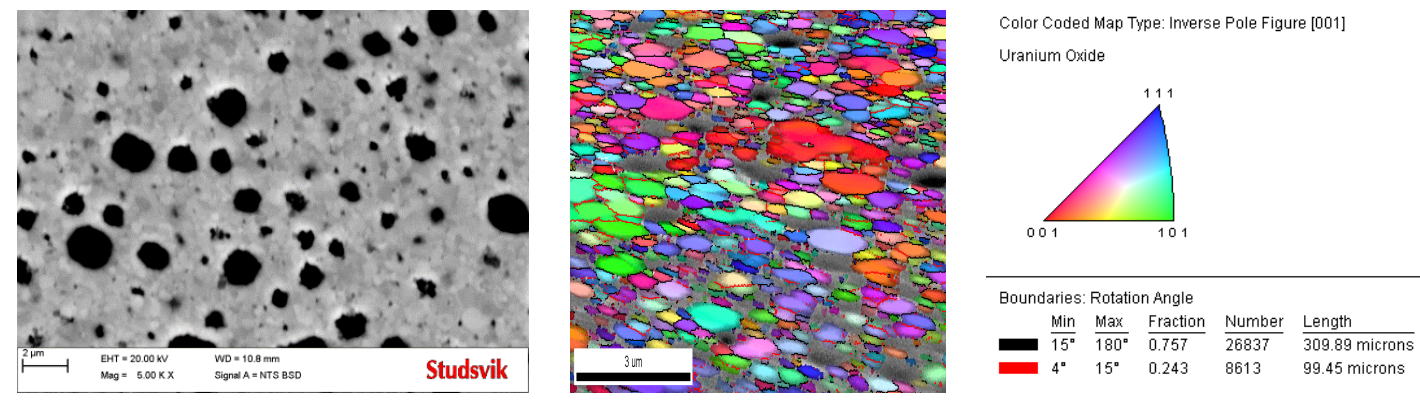

Figure 2. BSE micrograph resolving the small-grained structure in the rim region of the fuel (left), EBSD inverse pole figure map (middle) and color coding and grain boundary information (right).
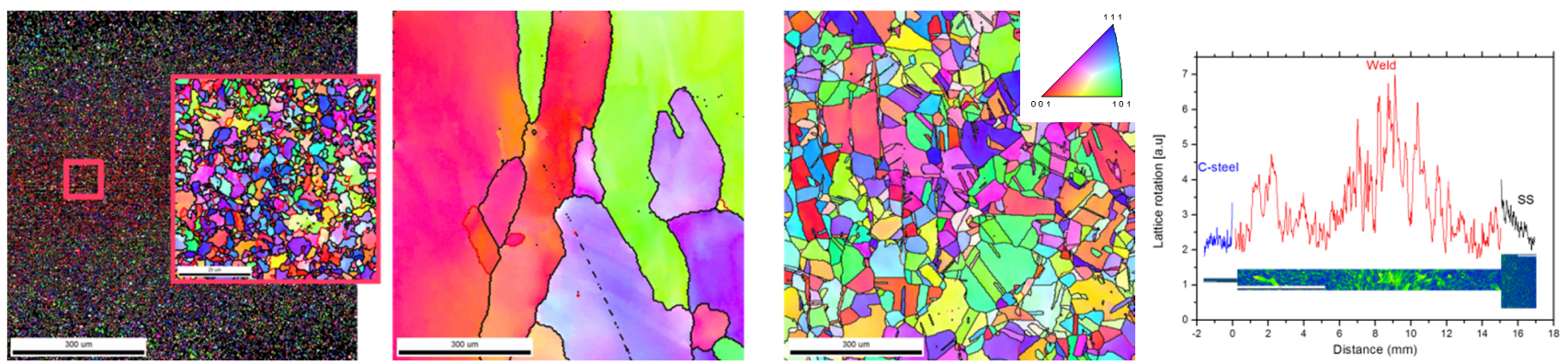

Figure 3. EBSD inverse pole figure maps of each region of the joint. The right graph shows the strain distribution across the weld and the corresponding EBSD misorientation maps. 